\title{
Estudo de validação de instrumento para mensurar resiliência em trabalhadores
}

\section{Instrument Validation Study to Measure Worker Resilience}

\author{
DAIANE NUNES \\ MARLEY ROSANA MELO DE ARAÚJO \\ Universidade Federal de Sergipe - UFS.
}

\section{RESUMO}

No contexto do trabalho, resiliência é entendida como a capacidade do sujeito de se adaptar e fortalecer mediante experiências de adversidade vivenciadas naquele ambiente. Tendo em vista a escassez de instrumentos para mensurar resiliência nesse cenário, este estudo objetivou validar a Escala de Resiliência em amostra de 600 trabalhadores de diferentes áreas profissionais. A análise dos dados foi executada por meio de Análise Fatorial Exploratória, extração dos coeficientes alfa de Cronbach e estatísticas descritivas para caracterizar a amostra. Foram encontrados dois fatores: 1 - Autodeterminação $(\alpha$ : 0,76): conjunto de comportamentos e habilidades que dotam o sujeito da capacidade de ser agente causal em relação ao cumprimento de seus objetivos e metas estabelecidas (oito itens); 2 - Autoeficácia ( $\alpha$ : 0,69): percepção do sujeito acerca de sua competência pessoal na resolução de tarefas e situações (10 itens). Assim, este estudo apresenta à comunidade científica um instrumental válido e fidedigno para uso em outras pesquisas e intervenções.

Palavras-chave: Escala de Resiliência. Trabalhadores. Análise Fatorial Exploratória. 


\section{Abstract}

In the context of work, resilience is understood as the ability of the subject to adapt and strengthen through experiences of adversity experienced in that environment. Considering the scarcity of instruments to measure resilience in this scenario, this study aimed to validate the Resilience Scale in a sample of 600 workers from different professional areas. Data analysis was performed using Exploratory Factor Analysis, extraction of Cronbach's alpha coefficients and descriptive statistics to characterize the sample. Two factors were found: 1 - Self-determination $(\alpha: 0.76)$ : set of behaviors and abilities that endow the subject with the capacity to be a causal agent in relation to the fulfillment of his goals and established goals (eight items); 2 - Self-efficacy ( $\alpha$ : 0.69): perception of the subject about his personal competence in the resolution of tasks and situations (10 items). Thus, this study presents to the scientific community a valid and reliable instrument for use in other researches and interventions.

Key words: Resilience Scale. Workers. Exploratory Factorial Analysis.

\section{INTRODUÇÃo}

O mundo do trabalho vivenciou profundas transformações a partir do final do século XX e início do século XXI, configurando uma nova formatação de mercados, difusão de inovações tecnológicas e organizacionais nas mais diversas cadeias produtivas, em escala mundial (CIMBALISTA, 2010; 2006). Na atualidade, o momento histórico tem se caracterizado por intensas mudanças em diferentes âmbitos. As rápidas transformações tecnológicas e de equacionamento econômico têm demandado das instituições e do indivíduo um processo de adaptação com a mesma intensidade e rapidez. Deste último, espera-se comportamentos que impliquem em flexibilidade de ação, de estrutura e de vida pessoal, como meio de ajustamento às novas contingências e condições. Assim, o desempenho profissional obriga o indivíduo a administrar esse aspecto de sua vida, de modo que o trabalho passa a ser uma reposição de si mesmo, já que as referências ao seu redor, das quais extrai valor e sentido para si mesmo, estão constantemente sofrendo alterações (BARLACH; LIMONGI-FRANÇA; MALVEZZI, 2008; FERNANDES, SIQUEIRA, VIEIRA, 2014; SANTOS; QUEL; VIEIRA; ROSINI, 2019). 
O trabalho, na sociedade atual, demanda do trabalhador maior uso de sua subjetividade para o enfrentamento de situações adversas, evidenciadas pelo ritmo intensificado da jornada e pelas imposições advindas do mercado. Essas transformações, no contexto laboral, geraram situações desgastantes, em um ambiente comprometido com a produção em ritmo intensificado, evidenciando elementos inerentes às condições de emprego e à subjetividade do trabalhador (CIMBALISTA, 2006; ESTANISLAU; CASTRO; VIEIRA; RESCH, 2012; FILENGA; VIEIRA, 2012).

O conceito de resiliência emerge nesse contexto como uma mudança de paradigma, saindo da perspectiva da fraqueza do indivíduo para um olhar sobre a capacidade de enfrentar situações adversas e que colocam em risco sua saúde e bem-estar, estimulando no trabalhador um constante processo de descobrir, em si mesmo, novas formas de recobrar suas forças, de se adaptar a situações de risco e esforços despendidos (CIMBALISTA, 2006).

Dada a centralidade do trabalho na vida dos sujeitos, e diante das novas condições impostas pelo mundo contemporâneo do trabalho, bem como considerando a dificuldade de conceituação do construto, a pesquisa sobre como ocorrem os processos de resiliência no cenário atual têm se revelado de fundamental importância. Para auxiliar nestas investigações, existem, na literatura científica, diferentes instrumentos com medidas válidas e confiáveis para avaliar o fenômeno resiliência, ainda que os mesmos adotem variadas concepções e entendimentos sobre o construto. Essa diversidade se justifica no fato do fenômeno ser ainda considerado um construto em aberto e de definição teoricamente pouco sólida. Destarte a diversidade de aparato teórico no qual estes instrumentos se assentam, impõe-se como fundamental a constante reavaliação das características psicométricas e checagem da estrutura empírica destas medidas em diferentes contextos, pois as distintas escalas podem vir a apresentar problemas de validade e confiabilidade, como divergências entre populações-alvo e culturas (ONÇA, 2011).

Tendo em vista este cenário e a parca cobertura do fenômeno resiliência voltado para o contexto do trabalho pelo aparato instrumental disponível, bem como o fato de que alguns instrumentos largamente aceitos pela comunidade científica internacional 
ainda não foram testados no Brasil em amostra de trabalhadores, o presente estudo teve como objetivo a validação, em amostra de trabalhadores, da Escala de Resiliência adaptada para o Brasil por Pesce et al. (2005), e originalmente (Resilience Scale) desenvolvida por Wagnild e Young (1993).

Cabe ressaltar que, grande parte dos trabalhos que se propuseram a estudar o fenômeno resiliência, tanto dentro quanto fora do contexto organizacional, o fizeram por meio de pesquisas de caráter qualitativo, de modo que este estudo se propôs a apresentar à comunidade de estudiosos e profissionais da área aplicada, um instrumental estruturado, válido e fidedigno, para uso em outras pesquisas e intervenções, a fim de trazer mais esclarecimentos sobre o tema.

\section{RESILIÊNCIA}

Os primeiros estudos com o intuito de conceituar resiliência datam da passagem da década de 1970 a 1980, quando pesquisadores americanos e ingleses passaram a observar o fenômeno dos indivíduos que permaneciam saudáveis, mesmo após serem expostos a situações adversas. Inicialmente, esse fenômeno foi denominado "invulnerabilidade" e esses indivíduos, chamados de invulneráveis. A partir de estudos posteriores, o termo invulnerabilidade foi substituído por resiliência, levando em consideração o fato de que o vocábulo "invulnerabilidade" implicava afirmar que os sujeitos que haviam sidos expostos a situações estressoras não sofriam os impactos adversos em sua saúde mental, sendo o termo resiliência considerado mais apropriado (BRANDÃO; MAHFOUD; GIANORDOLI-NASCIMENTO, 2011).

No processo de construção do conceito de resiliência, os estudos iniciais foram liderados por pesquisadores de diversas partes do mundo, sob diferentes perspectivas, posteriormente organizadas em três correntes: a norte-americana ou anglo-saxônica, a europeia e a latino-americana. A corrente norte-americana possui um direcionamento mais pragmático e centrado no indivíduo, com enfoque behaviorista, tomando para a avaliação da resiliência indicadores observáveis e quantificáveis. Para essa linha de pensamento, a resiliência surge como produto da interação entre o sujeito e o meio em que ele está inserido. A corrente europeia teria uma perspectiva 
mais relativista, pautando-se na Psicanálise e tomando a visão do sujeito como fundamental para o julgamento de resiliência. Para essa corrente, a resposta do sujeito às adversidades ultrapassa os fatores do meio e é produzida a partir da dinâmica psicológica do indivíduo. Por sua vez, a corrente latino-americana é mais comunitária, com enfoque no social como resposta aos problemas do sujeito em meio a situações adversas (BRANDÃO; MAHFOUD; GIANORDOLI-NASCIMENTO, 2011).

Na literatura brasileira, há um certo consenso quanto à atribuição da origem do conceito de resiliência psicológica às Ciências Exatas, especificamente, à Física. Grande parte dos pesquisadores no Brasil (COSTA; ASSIS, 2006; BELANCIERI, 2007; COUTO-OLIVEIRA, 2007; JUNQUEIRA; DESLANDES, 2003; LIBÓRIO; CASTRO; COÊLHO, 2006; POLETTO, 2007; POLETTO; KOLLER, 2006, 2008; SOUZA; CERVENY, 2006; YUNES, 2003; YUNES; MENDES; ALBUQUERQUE, 2005; YUNES; SZYMANSKI; TAVARES, 2001) apontam que o termo resiliência é originário do latim (resilio), que significa retornar a um estado anterior, significado utilizado nas Ciências Naturais, pela Engenharia e pela Física, para se referir à capacidade que um corpo tem de voltar ao seu estado normal após sofrer alterações sobre si.

No entanto, para as Ciências Humanas, especificamente a Psicologia, o conceito de resiliência precisou ser adaptado, visto que não se pode entender que um determinado indivíduo, tendo vivenciado situações adversas e estressoras, possa voltar à forma inicial (CARVALHO; TEODORO; BORGES, 2015). No que se refere ao ser humano, a resiliência não pode implicar em um retorno a um estado anterior, mas em uma superação ou adaptação frente a uma dificuldade compreendida como um risco, e a possibilidade de uma construção positiva e um processo de subjetivação mediante o enfrentamento de situações que implicam em consequências negativas para o sujeito (JUNQUEIRA; DESLANDES, 2003).

O construto resiliência é utilizado para explicar porque alguns indivíduos apresentam e revelam resultados psicológicos positivos, apesar de terem sofrido experiências negativas de risco (LUTHAR; CICCHETTI; BECKER, 2000; YUNES, 2003; CASTLEDEN; MCKEE, MURRAY; LEONARDI, 2011; BARLACH; LIMONGI-FRANÇA; MAL- 
VEZZI, 2008, LOPES; MARTINS, 2011; PESCE et al., 2005; GURGEL et al., 2013). Desse modo, pode ser definida como a capacidade do sujeito de se adaptar e manter seu funcionamento positivo frente às adversidades ou exposição a estressores extremos. Atualmente, o conceito de resiliência tem implicado a incorporação de elementos como ganhos de crescimento e de aprendizagem, que decorrem do enfrentamento de situações que se apresentam como fatores de risco para o bem-estar do sujeito (MARTINS, 2015).

Nesse sentido, o conceito de resiliência é entendido como um processo dinâmico que inclui variáveis que atuam como fatores de proteção à adversidade. Esses fatores incluem características do indivíduo, como a autoestima, autodeterminação, flexibilidade, habilidade para resolução de problemas, autoeficácia, entre outros; a coesão e o bom relacionamento intrafamiliar e; a disponibilidade de suporte externo que proporcione o encorajamento e reforce as estratégias de enfrentamento dos sujeitos, suporte este que pode ser proveniente do grupo de pares e comunidade (PESCE et al., 2005)

No contexto de trabalho contemporâneo, pode-se entender o fenômeno da resiliência como a construção de soluções e atitudes criativas diante dos obstáculos impostos por este ambiente. Em outras palavras, a resiliência no ambiente organizacional se refere à existência ou construção de recursos adaptativos, que possibilitam a preservação do bem-estar e a relação saudável entre o ser humano e o seu trabalho, em um ambiente em constante transformação e marcado por diversas formas de ruptura (BARLACH; LIMONGI-FRANÇA; MALVEZZI, 2008).

O trabalho flexibilizado exige atuação flexível do trabalhador, esperando que o mesmo se mostre polivalente, multifuncional, engajado no processo de trabalho e na cultura da empresa, demonstrando capacidade para lidar com processos cada vez mais dinâmicos. Desse modo, o trabalhador, no contexto atual, se sujeita aos estilos de gestão, suporta o ritmo intensificado, as pressões e responsabilidade (RIBEIRO et al., 2011). Essas constantes mudanças trazem consigo diferentes formas de ruptura e crises que refletem diretamente sobre a saúde do trabalhador, demandando deste muito esforço para o ajustamento de sua identidade à metamorfose permanente eviden- 
ciada no mundo do trabalho (MALVEZZI, 2000).

Diante do exposto, destaca-se a relevância de estudos sobre como ocorrem os processos de resiliência no cenário atual, em específico no contexto de trabalho, e como se dá a dinâmica entre os fatores de risco - potencializadores de elementos que provocam adoecimento de sujeitos expostos a condições adversas, provenientes das novas formatações do trabalho contemporâneo -, e os fatores protetivos, que minimizam o impacto dessas situações de risco para a saúde do trabalhador (RIBEIRO et al., 2011).

No que se refere à mensuração e estudos empíricos que abarcam o construto resiliência, localizam-se na literatura diferentes instrumentos com medidas válidas e confiáveis para avaliar o fenômeno e que adotam diferentes concepções e modelos teóricos. Essa diversidade se justifica no fato do fenômeno ser ainda considerado um construto em aberto e de definição teoricamente pouco sólida (ONÇA, 2011).

No entanto, cabe ressaltar que, embora seja crescente o interesse pelo conceito de resiliência, ainda são poucos os instrumentos validados e disponíveis para avaliar esse construto. Nesse sentido, Gurgel et al. (2013) buscaram, através de uma revisão sistemática, identificar instrumentos válidos utilizados na literatura nacional e internacional para avaliar resiliência em adultos e idosos. Os resultados desta iniciativa apontaram dezesseis (16) estudos que validaram treze (13) instrumentos diferentes com a função de avaliar resiliência, entre eles: The Brief Resilience Scale (BRS), validada por Smith et al. (2008); The Connor-Davidson Resilience Scale (CD-RISC), validada por Connor e Davidson (2003); 10-item CD-RISC, validada por Campbell-Sills e Stein (2007); The Resilience Scale for Adults (RSA), validada por Friborg et al. (2003); Brief Resilient Coping Scale, validada por Sinclair e Wallston (2004); Adolescent Resilience Scale, validada por Oshio et al. (2003); Deployment Risk and Resilience Inventory (DRRI), validada por King et al. (2006) e a Resilience Scale, validada para o Brasil por Pesce et al. (2005) e desenvolvida originalmente por Wagnild e Young (1993).

O estudo conduzido por Piacentini (2014) aponta para a existência de alguns instrumentos de mensuração de resiliência validados para o contexto brasileiro, entre eles: a) o Questionário do Índice 
de Resiliência de Reivichi e Shatté (2002), adaptado por Barbosa (2006), apresentando um modelo de sete fatores (administração das emoções, controle dos impulsos, otimismo com a vida, análise do ambiente, empatia, autoeficácia e alcançar pessoas), composto por 56 itens; b) a Escala de Resiliência Connor-Davidson, desenvolvida por Connor e Davidson (2003), revalidada por análise fatorial confirmatória por Campbell-Sills e Stein (2007), cunhada como CD - RISC-10, e adaptada por Lopes e Martins (2011), sendo a versão adaptada de caráter unidimensional; c) a Escala Pilares da Resiliência, desenvolvida por Cardoso e Martins (2013), apresentando um modelo de onze fatores (autoeficácia, controle emocional, autoconfiança, orientação positiva para o futuro, valores positivos, empatia, reflexão, sociabilidade, aceitação positiva de mudança, independência e bom humor), reunindo 90 itens; d) a Escala de Resiliência, uma das mais utilizadas no Brasil, desenvolvida originalmente por Wagnild e Young (1993), e adaptada para o contexto nacional por Pesce et al. (2005), disponibilizando para a comunidade científica uma estrutura com três fatores (Resolução de problemas e valores; Independência e determinação e; Autoconfiança e capacidade de adaptação a situações), resguardando os 25 itens originais e, por último; e) a Escala de Resiliência-14, de Wagnild e Young (2010), adaptada por Damásio, Borsa e Silva (2011), com modelo unifatorial.

No contexto organizacional e do trabalho, essa problemática é ainda maior, uma vez que foram localizados poucos estudos de resiliência com medidas validadas no Brasil em amostra de trabalhadores (CARVALHO et al., 2014; HJEMDAL et al., 2009; LOPES; MARTINS, 2011). Desse modo, diante do exposto, observa-se a carência de um aparato instrumental para mensurar resiliência no contexto do trabalho, bem como o fato de que alguns instrumentos largamente aceitos pela comunidade científica internacional ainda não foram testados no Brasil em amostra de trabalhadores.

Portanto, o presente estudo teve como objetivo a validação, em amostra de trabalhadores, da versão da Escala de Resiliência adaptada para o Brasil por Pesce et al. (2005), e originalmente (Resilience Scale) desenvolvida por Wagnild e Young (1993). Além disso, buscou comparar a estrutura fatorial revelada no presente estudo com aquela derivada da validação do instrumento com amostra de 
estudantes (PESCE et al., 2005) e com os resultados do estudo original em amostra de idosos norte-americanos (WAGNILD; YOUNG, 1993), a fim de verificar a estabilidade do construto.

A Escala de Resiliência foi desenvolvida a partir de um estudo qualitativo com 24 mulheres adultas, previamente selecionadas por apresentarem adaptação com sucesso a adversidades da vida. Foi solicitado que cada uma descrevesse como se organizava diante de vivências negativas e, a partir de suas narrativas, foram identificados cinco componentes hipotéticos ilustrativos de resiliência: serenidade, perseverança, autoconfiança, sentido de vida e auto-suficiência (WAGNILD; YOUNG, 1993).

Após a elaboração da medida, Wagnild e Young (1993) conduziram análise fatorial exploratória com amostra de 810 idosos, onde foram encontrados dois fatores denominados "Competência pessoal", que incluiu 17 itens com ideias de autoconfiança, independência, determinação, invencibilidade, controle, desenvoltura e perseverança; e "Aceitação de si mesmo e da vida", que agrupou 8 itens com ideias de adaptabilidade, equilíbrio, flexibilidade e perspectiva de vida equilibrada. O coeficiente alfa de Cronbach da medida foi equivalente a 0,91 , apresentando boa consistência interna.

No contexto brasileiro, Pesce et al. (2005) realizaram uma adaptação transcultural e avaliação psicométrica da escala de resiliência desenvolvida por Wagnild e Young (1993). A amostra do estudo consistiu de 997 escolares de $7^{\underline{a}}$ e $8^{\underline{a}}$ séries do ensino fundamental e $1^{\circ}$ e $2^{\circ}$ anos do ensino médio da rede pública de ensino do município de São Gonçalo, no Rio de Janeiro. Os autores pretenderam, através de análise comparativa entre o instrumento original e sua versão para o português, contribuir para a avaliação transcultural do construto.

Na versão validada por Pesce et al. (2005), os autores optaram por manter no estudo os fatores que explicassem a variância total em mais de 5\%. Assim, foram encontrados três fatores, que explicaram 32,8\% da variância na rotação Oblimin. O primeiro fator encontrado pelos autores explicou $20,6 \%$ da variância total e abarcou itens que indicam resolução de ações e valores que dão sentido à vida. O segundo fator, explicou 6,7\% da variância total e incluiu itens que expressavam ideia de independência e deter- 
minação. O terceiro e último fator agrupou itens indicativos de autoconfiança e capacidade de adaptação a situações, explicando $5,5 \%$ da variância total.

Esta escala consiste em uma das poucas usadas para medir níveis de adaptação psicossocial positiva frente a eventos importantes de vida. Desse modo, o presente estudo pretende munir a comunidade de estudiosos e profissionais da área organizacional e do trabalho com um instrumental válido e fidedigno para uso em outras pesquisas e intervenções, trazendo mais esclarecimentos sobre o fenômeno resiliência no contexto de trabalho.

\section{Método}

\section{Amostra}

A amostra da presente pesquisa foi constituída por 600 trabalhadores de diferentes profissões e ramos de negócio, maiores de dezoito anos que, atualmente, estivessem empregados formalmente. Foi adotada a amostragem por conveniência, e a participação voluntária e anônima.

A média de idade dos participantes foi de 31,8 anos (DP $=$ 10,4), com idade mínima de 18 anos e máxima de 68 anos, em sua maioria compostos pelo sexo masculino (53,5\%), enquanto que o sexo feminino representou $46,5 \%$ da amostra. No que se refere à escolaridade, 48,8\% dos participantes tinham nível médio ou abaixo, enquanto que $51,2 \%$ superior ou acima.

Com relação aos dados profissionais, o setor econômico predominante foi "comércio e serviços" (90,5\%) e 56,5\% da amostra exercia função em empresas privadas. A faixa salarial dos respondentes teve mediana de $\mathrm{R} \$ 1.000,00$, com menor valor relatado de $\mathrm{R} \$ 200,00 \mathrm{e}$ maior valor de $\mathrm{R} \$ 11.245,00$. Cumpre ressaltar, ainda, que o tempo de trabalho no emprego atual teve mediana de dois anos, variando de um mês a quarenta anos. 
Tabela 1 - Caracterização da amostra

\begin{tabular}{llll}
\hline Variáveis & Categorias & $\begin{array}{l}\text { Frequência } \\
\text { Absoluta }\end{array}$ & $\begin{array}{l}\text { Frequência } \\
\text { Relativa }\end{array}$ \\
\hline Sexo & Masculino & 321 & $53,5 \%$ \\
& Feminino & 279 & $46,5 \%$ \\
\hline Escolaridade & $\begin{array}{l}\text { Ensino Fundamental } \\
\text { ou Médio }\end{array}$ & 293 & $48,8 \%$ \\
& $\begin{array}{l}\text { Ensino Superior ou } \\
\text { Tipo da Empresa }\end{array}$ & 307 & $51,2 \%$ \\
& Pós-graduaçáo & & \\
\hline \multirow{2}{*}{ Setor Econômico } & Privada & 261 & $43,5 \%$ \\
& Indústria & 339 & $56,5 \%$ \\
\hline & Comércio e Serviços & 535 & $9,1 \%$ \\
& & & $90,5 \%$ \\
\hline
\end{tabular}

\section{INSTRUMENTOS}

Os instrumentos utilizados incluem um questionário sociodemográfico e a Escala de Resiliência adaptada para o Brasil por Pesce et al. (2005), e originalmente desenvolvida por Wagnild e Young (1993). O processo de adaptação transcultural da escala contou com procedimento de validação qualitativa proposto por Herdman, Fox-Rushby e Badia (1998 citado por Pesce et al., 2005), que consiste na realização de uma série de passos rigorosos a serem seguidos até a aceitação de equivalência entre os instrumentos. Esse procedimento inclui, entre outros aspectos, a avaliação de especialistas na área, a fim de verificar se o domínio do instrumento original é pertinente ao conhecimento sobre resiliência. Desta forma, não se evidenciou a necessidade de realização de novo processo de validação qualitativa para o uso da escala nessa amostra. A Escala de Resiliência contém 25 itens descritos de forma positiva, com respostas tipo Likert variando entre 1 (discordo totalmente) a 7 (concordo totalmente). Os escores da escala oscilam entre 25 a 175 pontos, com valores altos indicando elevada resiliência. Utilizou-se também um questionário sociodemográfico que continha informações pessoais como sexo, idade, escolaridade, dados profissionais como profissão/ocupação, o tempo de trabalho na atual empresa, o cargo ocupado, salário, etc. 


\section{Aspectos Éticos}

Este projeto foi aprovado pelo Comitê de Ética em Pesquisa com Seres Humanos do Hospital Universitário da Universidade Federal de (omitido para análise), sob o protocolo CAAE 51142215.7.0000.5546. Ao coletar a anuência do participante em ser voluntário na pesquisa, foi solicitada a assinatura do Termo de Consentimento Livre e Esclarecido (TCLE), contendo o objetivo da pesquisa, critérios de inclusão, as instruções da atividade a ser desenvolvida, as garantias de sigilo, de anonimato, de direito de recusa ou retirada do consentimento em qualquer fase do processo de pesquisa, os benefícios e riscos prováveis da mesma e o contato da pesquisadora para eventuais esclarecimentos e divulgação dos resultados da pesquisa.

\section{Procedimentos para coleta dos dados}

Os dados da pesquisa foram coletados de forma presencial, individual, auto aplicada, com previsão estimada de vinte minutos para o preenchimento dos instrumentos. Os participantes foram abordados por conveniência em suas residências, locais de trabalho, instituições de ensino e logradouros públicos. Durante a aplicação dos instrumentos, explicaram-se aos respondentes os objetivos da pesquisa, as instruções de preenchimento dos instrumentos e foram, ainda, retiradas as dúvidas levantadas.

\section{Procedimentos para análise dos dados}

Para análise dos dados, foi utilizado o programa estatístico IBM SPSS, versão 20.0. Realizaram-se análises exploratórias para correção de dados faltosos, erros de digitação e casos extremos (outliers), com objetivo de atender aos pressupostos para a utilização de estatísticas multivariadas.

Executou-se Análise Fatorial Exploratória (AFE) por meio de extração por Componentes Principais na solução inicial, para estimar o número de fatores e fatorabilidade da matriz de correlações; extração por Eixos Principais com rotação Promax, e carga fatorial acima de 0,30, recomendado pela literatura (DAMÁSIO, 2012). Os índices de fidedignidade utilizados foram o alfa de Cronbach $(\alpha)$ e a Confiabilidade Composta (CC), sendo este útlimo obtido através 
da calculadora digital do site The Statistical Mind (COLWELL, 2016). Adicionalmente, foram realizadas estatísticas descritivas para a caracterização da amostra nas variáveis sócio-demográficas e quanto aos escores nos fatores.

\section{RESULTADOS}

O processo de validação da Escala de Resiliência implicou algumas etapas estatísticas, a fim de verificar a estrutura fatorial e fidedignidade da mesma. Portanto, o primeiro passo durante a implementação da análise fatorial exploratória foi observar se a matriz de dados era passível de fatoração, isto é, analisar se os dados poderiam ser submetidos a esse processo. Os dados se mostraram adequados para análise fatorial exploratória, baseando-se nos critérios de Kaiser-Meyer-Olkin $(\mathrm{KMO}=0,82)$, e do Teste de Esfericidade de Bartlett $\left(\chi^{2}=2654,902 ; \mathrm{p}<0,001\right)$.

Demonstrada a fatorabilidade da Escala de Resiliência, foi realizada análise fatorial exploratória, que visa identificar a proximidade entre os itens de um instrumento, através de padrões de correlações estabelecidos entre eles (DANCEY; REIDY, 2013). Define-se Análise Fatorial Exploratória (AFE) como um conjunto de técnicas multivariadas que tem por objetivo principal encontrar a estrutura subjacente em dada matriz de dados e determinar o número e a natureza das chamadas variáveis latentes ou fatores que melhor representam um conjunto de variáveis observadas. Assim, as variáveis observadas fazem parte de um mesmo fator quando, e se, elas partilham uma variância comum. A AFE é conduzida, geralmente, quando o pesquisador não possui uma teoria prévia ou evidências empíricas necessárias para explicar como os itens de determinado instrumento devem ser agrupados e avaliados, ou quando o pesquisador pretende confirmar ou refutar dada estrutura fatorial de determinado instrumento. Assim, durante os procedimentos realizados de uma AFE, é necessário que todas as decisões sejam pautadas em critérios teóricos e metodológicos claros, com objetivo de obter modelos fatoriais adequados (DAMÁSIO, 2012).

Neste estudo, a solução inicial foi pedida por meio de extração dos Componentes Principais (Principal Component Analysis - PC), a qual demonstrou a possibilidade de retenção de até 8 fatores pelo 
critério de eigenvalue > 1 (KAISER, 1960), totalizando 55,44\% de variância explicada. O gráfico Scree Plot (CATTEL, 1966), por sua vez, sinalizava a possibilidade de existência de até 2 fatores. A análise paralela (HORN, 1965) acusava a possibilidade de acolher 4 fatores.

Procedeu-se, então, várias tentativas de solução fatorial, utilizando extrações por componentes principais (PC) ou por eixos principais (PAF), rotações ortogonais ou obliquas, imputação de 2 a 3 fatores, carga fatorial mínima para a retenção do item variando de 0,30 a 0,40 . Tais procedimentos de análise de dados objetivavam obter a solução mais parcimoniosa e de melhor interpretabilidade. A solução definitiva utilizou extração por eixos principais (PAF), rotação oblíqua Promax e critério de carga fatorial acima de 0,30, produzindo dois fatores que explicaram $19,9 \%$ da variância total do instrumento, valor este que corrobora com os resultados geralmente encontrados em pesquisas da área de humanas e, em especial, da Psicologia. A correlação entre os dois fatores extraídos foi moderada $(\mathrm{r}=0,65)$, sinalizando para a interrelação de seus conteúdos e a confiabilidade da escala $(\alpha=0,76)$ alcançou as exigências psicométricas para ser considerada uma medida consistente. Adicionalmente, o resultado da confiabilidade composta $(0,82)$ também indicou boas propriedades psicométricas da Escala de Resiliência. A distribuição dos itens em cada fator, bem como as cargas fatoriais podem ser visualizadas na Tabela 2.

O primeiro fator foi chamado de "Autodeterminação" e agrupou oito itens, sendo definido como um conjunto de comportamentos e habilidades que dotam o sujeito da capacidade de ser agente causal em relação ao cumprimento de seus objetivos e metas estabelecidas. Este conteúdo está representado por itens como: item 15 - “Eu mantenho interesse nas coisas" e item 10 - "Eu sou determinado". O segundo fator, denominado "Autoeficácia", compreendeu dez itens da escala e se refere à percepção do sujeito acerca de sua competência pessoal na resolução de tarefas e situações. Este conteúdo está representado por itens como: item 5 - "Eu posso estar por minha conta se eu precisar" e; item 17 - "Minha crença em mim mesmo me leva a atravessar tempos difíceis". A Tabela 3 apresenta, dentre outras informações, os coeficientes de confiabilidade e variância explicada para cada fator, adicionalmente aos escores descritivos destes fatores. 
Tabela 2 - Matriz de cargas fatoriais

Item

Carga

Fatorial

\section{FATOR 1 - Autodeterminação}

15. Eu mantenho interesse nas coisas.

0,73

14. Eu sou disciplinado.

0,68

1. Quando eu faço planos, eu levo eles até o fim.

0,57

10. Eu sou determinado.

0,56

4. Manter interesse nas coisas é importante para mim

0,40

6. Eu sinto orgulho de ter realizado coisas em minha vida.

0,37

24. Eu tenho energia suficiente para fazer o que eu tenho que fazer.

0,36

21. Minha vida tem sentido.

0,35

\section{FATOR 2 - Autoeficácia}

5. Eu posso estar por minha conta se eu precisar.

3. Eu sou capaz de depender de mim mais do que de qualquer outra pes-

0,52 soa.

0,48

17. Minha crença em mim mesmo me leva a atravessar tempos difíceis.

0,42

25. Tudo bem se há pessoas que não gostam de mim.

0,40

16. Eu normalmente posso achar motivo para rir.

2. Eu costumo lidar com os problemas de uma forma ou de outra.

18. Em uma emergência, eu sou uma pessoa com quem as pessoas podem contar.

19. Eu posso geralmente olhar uma situação de diversas maneiras.

0,33

9. Eu sinto que posso lidar com várias coisas ao mesmo tempo.

13. Eu posso enfrentar tempos difíceis porque já experimentei dificuldades antes.

Tabela 3 - Propriedades psicométricas da Escala de Resiliência.

\begin{tabular}{l|l|l|l|l|l|l}
\hline FATORES & DEFINIÇÃO & $\mathrm{r}^{2}$ & $\alpha$ & $\mathrm{M}$ & $\mathrm{DP}$ & ITENS \\
\hline $\begin{array}{l}\text { FATOR 1- Auto- } \\
\text { determinação }\end{array}$ & $\begin{array}{l}\text { Conjunto de comporta- } \\
\text { mentos e habilidades que } \\
\text { dotam o sujeito da capaci- } \\
\text { dade de ser agente causal } \\
\text { em relação ao cumprimento } \\
\text { de seus objetivos e metas } \\
\text { estabelecidas. }\end{array}$ & $16,6 \%$ & 0,76 & 5,74 & 0,78 & $\begin{array}{l}15,14,1, \\
10,4,6,24, \\
21 .\end{array}$ \\
\hline $\begin{array}{l}\text { FATOR 2- } \\
\text { Autoeficácia }\end{array}$ & $\begin{array}{l}\text { Percepção do sujeito acerca } \\
\text { de sua competência pessoal } \\
\text { na resolução de tarefas e } \\
\text { situações. }\end{array}$ & $3,3 \%$ & 0,69 & 5,45 & 0,76 & $\begin{array}{l}5,3,17,25, \\
16,2,18, \\
19,9,13 .\end{array}$ \\
\hline
\end{tabular}

Desse modo, a escala que, anteriormente, tinha 25 itens, foi reduzida a 18 itens em sua totalidade, observando a carga fatorial mínima exigida $(0,30)$ para retenção dos itens. Foram excluídos os itens $23,12,8,11,22$, 7 e 20, convergindo, assim, para a homoge- 
neidade da medida e sua parcimônia, e contribuindo na rapidez de aplicação da escala e aderência dos participantes ao estudo.

Com relação à análise de fidedignidade dos fatores, observamos que ambos apresentaram boa consistência interna: o fator "Autodeterminação" apresentou coeficiente alfa de Cronbach equivalente a 0,76; enquanto que o fator "Autoeficácia" apresentou $\alpha=0,69$. Segundo Damásio (2012), o alfa de Cronbach avalia o grau em que os itens de uma matriz de dados estão correlacionados entre si. Em sua totalidade, a escala apresentou escore de fidedignidade $\alpha=0,76$, demonstrando assim, bom indicativo de correlação subjacente entre todos os itens, sinalizando para a homogeneidade da medida.

No que se refere aos escores médios em cada fator, Autodeterminação teve média de 5,74 (DP =0,78), demonstrando que os participantes reconhecem sua capacidade de determinar o alcance de resultados desejados. O fator Autoeficácia, por sua vez, denota que a amostra tende a perceber-se capaz de resolver tarefas e situações (M $=5,45$; $\mathrm{DP}=0,76$ ), uma vez que seu escore médio alcançou pontuação localizada entre as alternativas de resposta "Concordo um pouco" e "Concordo". Conjuntamente, os escores nos fatores da escala alcançam valores indicativos de resiliência por parte da amostra.

\section{DISCUSSÃo}

Nesta seção, serão inicialmente apresentadas, de forma sintetizada, as descobertas dos diferentes estudos (PESCE et al., 2005; WAGNILD; YOUNG, 1993), comparativamente aos resultados obtidos a partir desta pesquisa. Posteriormente, serão discutidos os principais aspectos nos quais eles diferem e se assemelham. Além disso, serão discutidos os resultados decorrentes do presente estudo, quanto à Análise Fatorial Exploratória e às análises descritivas.

Neste estudo, a partir da AFE, foram extraídos dois fatores denominados Autodeterminação (oito itens) e Autoeficácia (dez itens), conforme apresentado na seção Resultados. No estudo original, desenvolvido pelos autores americanos Wagnild e Young (1993), com amostra de 810 idosos, foram encontrados dois fatores, denominados "Competência pessoal" (17 itens - sugere autoconfiança, independência, determinação, invencibilidade, controle, desenvoltura e perseverança) e "Aceitação de si mesmo e da vida" (8 itens 
- representa adaptabilidade, equilíbrio, flexibilidade e perspectiva de vida equilibrada).

Por sua vez, no estudo de adaptação transcultural da escala para o Brasil, Pesce et al. (2005) abdicaram da classificação original, ao interpretar o resultado de sua análise fatorial da seguinte maneira: o primeiro fator reuniu 14 itens que indicam resolução de ações e valores, por exemplo, amizade, realização pessoal, satisfação, etc.; o segundo fator agrupou 4 itens que transmitem a ideia de independência e determinação; no terceiro fator, por sua vez, encontram-se 6 itens indicativos de autoconfiança e capacidade de adaptação a situações.

No que se refere aos aspectos conceituais do fenômeno resiliência expressos nos estudos, pode-se observar que as três configurações, apesar de apresentarem disposições de fatores e nomenclaturas distintas, como apontado acima, denotam também algumas similaridades entre si. Destaca-se que nos três estudos foram encontrados elementos que expressam ideia de independência e determinação, bem como autoconfiança e autoeficácia - fatores 2 e 3 em Pesce et al. (2005); fator 1 em Wagnild e Young (1993) e; fatores 1 e 2 no presente estudo. Nesse sentido, entendendo o construto resiliência enquanto um processo dinâmico, Onça (2011) aponta que competências pessoais como autonomia, comportamento direcionado para metas, habilidades para resolver problemas, autodeterminação, entre outros, somadas a aspectos familiares e redes de apoio do ambiente, são apontados como elementos na promoção de resiliência. Esta convergência de resultados pode apontar para o fato de que, embora a organização das estruturas fatoriais tenha apresentado diferenças em número e composição de fatores, conceitualmente os significados encerrados em tais estruturas não são tão distantes, o que colabora para a paulatina aproximação de uma definição mais consistente sobre o que seja resiliência.

Continuando com o exercício de comparação entre os estudos, na análise empreendida por Wagnild e Young (1993), optou-se pela rotação oblíqua, carga fatorial 0,40 para retenção do item, gerando cinco fatores como solução. No entanto, ao aplicar o critério de extrair fatores que congregassem, no mínimo, 5\% de variância explicada, dois fatores foram encontrados e provaram-se mais interpretáveis (Competência pessoal; Aceitação de si mesmo e da vida). 
No estudo de Pesce et al. (2005), similarmente à decisão de Wagnild e Young, os autores optaram por manter os fatores que explicassem a variância total em mais de 5\%. Com esse critério, eles encontraram três fatores que explicaram $32,8 \%$ da variância, quando utilizada rotação Oblimim. Utilizando o método da rotação ortogonal Varimax, o mesmo número de fatores foi encontrado, explicando 39,9\% da variância. Ao comparar os dois resultados, os autores observaram que o primeiro fator se manteve substancialmente intacto, tanto na rotação Oblimim quanto na Varimax, com algumas exceções. Já os demais fatores se mostraram mais híbridos. Entre as duas soluções possíveis neste estudo, os autores optaram pela versão em rotação ortogonal Varimax.

No presente estudo, procurou-se adotar, durante a AFE, os mesmos procedimentos utilizados por Pesce et al. (2005). Porém, decidiu-se manter a solução com rotação oblíqua Promax, tendo em vista sua melhor interpretabilidade e o fato de que a literatura ressalta que os resultados obtidos por meio do método Varimax, bem como por meio de todos os métodos ortogonais, nas pesquisas em Psicologia, tendem a ser incoerentes, levando em consideração que as rotações ortogonais assumem que os fatores extraídos são independentes uns dos outros, divergindo das teorias psicológicas, que consideram a complexidade e interrelação dos fenômenos. As rotações oblíquas, por sua vez, permitem as correlações entre os fatores. Dentre os vários métodos existentes neste tipo de rotação, optou-se pelo método Promax, observando que não existe um método mais adequado que o outro (DAMÁSIO, 2012).

Foram observadas algumas diferenças nos resultados quanto à variância explicada dos estudos. Na AFE, a variância explicada refere-se à porção de variância comum que um ou mais fatores consegue extrair de um determinado conjunto de dados (DAMÁSIO, 2012). No estudo de Pesce et al. (2005), foi alcançada 32,8\% de variância explicada pela estrutura fatorial extraída, enquanto no presente estudo, a variância diminuiu $\left(r^{2}=19,9 \%\right)$. Tais diferenças podem ser justificadas, em parte, considerando o método de rotação adotado na análise, dado que o método de rotação ortogonal, utilizado por Pesce et al. (2005), tende a superestimar a variância. Observam-se ainda, diferenças na constituição da amostra entre os 
dois estudos: nesta pesquisa, a amostra se apresentou de forma mais heterogênea, formada por trabalhadores de diferentes categorias profissionais, vinculação (instituição pública $X$ iniciativa privada) e idades, comparativamente ao estudo transcultural de adaptação da escala com adolescentes, os quais constituíam amostra internamente mais homogênea. $O$ fato de a amostra de trabalhadores utilizada ser de composição mais heterogênea pode produzir um impacto na variância comum, dispersando-a entre subgrupos de trabalhadores provavelmente existentes na amostra, e contribuindo para o efeito final de redução da variância explicada pelos dados. Destarte, o resultado observado não contraindica o uso da escala na população de trabalhadores, mas pontua a necessidade de mais estudos comparativos entre grupos específicos de trabalhadores quanto à estrutura fatorial da Escala de Resiliência. Ademais, não há indicativo de "pontos de corte" determinados para o que seria um nível de variância explicada aceitável ou não aceitável, bem como se destaca que a sua porcentagem não deve ser utilizada como um indicador de importância para a interpretação de uma AFE (DAMÁSIO, 2012).

As decisões metodológicas no que concerne ao tipo de extração, de rotação e imputação de número de fatores são acompanhadas, em específico no estudo de Pesce et al. (2005), por algumas falhas quanto à ausência de critério de carga fatorial mínima exigida, tendo estas oscilado de 0,08 a 0,82 nos resultados destes autores. Indica-se como exemplo desta situação, os itens $7(0,08)$ e $12(0,17)$ no Fator 1 ; os itens $5(0,14)$ e $25(-0,10)$ no Fator 2 e; os itens $3(0,17)$ e $22(0,11)$ no Fator 3, os quais não atingiram a carga fatorial mínima de 0,30 em nenhum dos fatores, demonstrando a fragilidade da relação existente entre esses itens e o fator correspondente na estrutura interna destes autores. Além disso, se observado o pressuposto da carga fatorial mínima de 0,30, o Fator 2, apresentado pelos autores, permaneceria apenas com dois itens, invalidando sua existência.

A esse respeito, a literatura aponta que variáveis que apresentam dados muito díspares tendem a apresentar resultados empobrecidos, isto é, devem ser retiradas dos fatores por não atingirem carga fatorial mínima. Nesse sentido, ressalta-se que são comumente aceitas pela comunidade científica, cargas fatoriais que expressam valores em ponto de corte igual ou maior do que 0,30 (DAMÁSIO, 
2012). Portanto, como já apontado, a estrutura fatorial proposta por Pesce et al. (2005) apresenta algumas falhas no que tange a esses aspectos e precisa ser reconsiderada. Essa situação denota fragilidade na estrutura fatorial apresentada pelos referidos autores e dificulta a comparação entre seu estudo e o presente trabalho.

No que se refere aos fatores encontrados no presente estudo, decorrentes da AFE empreendida, a literatura tem apontado algumas relações existentes entre Autodeterminação, Autoeficácia e Resiliência. A autodeterminação representa um conjunto de comportamentos e habilidades que atribuem ao sujeito a capacidade de ser agente causal em relação ao seu futuro, em outras palavras, apresentar comportamentos intencionais. A teoria da autodeterminação foi elaborada por Ryan e Deci (1981) e tem como objeto de estudo as condições sociais e ambientais que promovem saúde mental, e como hipótese, a noção de que bem-estar psicológico pode ser alcançado a partir da autodeterminação (APPEL-SILVA; WENDT; ARGIMON, 2010). Esse conceito, assim como a noção de resiliência, insere-se numa perspectiva que perpassa a Psicologia Positiva, que pretendia desviar da tendência predominantemente clínica de focar as investigações no adoecimento, e redirecionar o olhar psicológico para um foco nas potencialidades e qualidades humanas (ONÇA, 2011).

A Autoeficácia, por sua vez, é um fenômeno descrito como conjunto de crenças do sujeito acerca da sua própria capacidade de organizar e executar determinado curso de ação para alcançar determinado resultado (BANDURA, 1977; 1997). Nesse sentido, Bandura (1986) estabelece relação entre crenças de autoeficácia e resiliência, apoiando-se na ideia de agência, autorregulação e sistema de crenças de autoeficácia, desenvolvidos pela teoria social cognitiva. Com a ideia de agência, Bandura (1986) explica que, como agente, o sujeito exerce papel ativo na sua existência e influencia o próprio comportamento e as circunstâncias de sua vida, pois não é apenas alvo das ações do meio, mas também age sobre ele e produz influências que o modificam (BANDURA, 1997; 1986; 1977 apud FONTES; AZZI, 2012). Portanto, as crenças de autoeficácia exercem papel fundamental para determinação do comportamento humano. Sendo assim, tais crenças atuam como facilitadores e mesmo atenuantes das diversas pressões advindas do meio ambiente, dentre 
elas, as hostis e desvantajosas para a adaptação. A forma como os sujeitos enfrentarão tais estressores vai determinar sua capacidade de adaptação e recuperação, isto é, sua resiliência. Daí a relevância do papel ativo das crenças de autoeficácia na promoção de resiliência (FONTES; AZZI, 2012).

Cabe ressaltar que os resultados do estudo conduzido, de validação da escala em amostra de trabalhadores, ainda não resolvem a problemática em torno da constituição do construto resiliência, mas apresenta algumas possibilidades na busca por solidificar o tema e aponta algumas possíveis saídas, ou mesmo, denota e reafirma a sua complexidade enquanto fenômeno da Psicologia.

Referente à problemática da carência de medidas específicas para resiliência no trabalho, Gomide Júnior, Silvestrin e Oliveira (2015) relatam que algumas medidas gerais de resiliência, transpostas para o contexto organizacional, têm sido utilizadas em trabalhos no Brasil (CARVALHO et al., 2014; HJEMDAL et al., 2009), mas são raros os estudos voltados para o construto resiliência no trabalho com o uso de medidas específicas para tal. Entre estes, foi encontrado apenas o estudo realizado por Lopes e Martins (2011), que validaram a escala CD-RISC 10 para o Brasil. Cumpre ressaltar que o presente estudo também trata do uso de uma medida geral de resiliência em amostra de trabalhadores, não eliminando ainda essa lacuna em relação à ausência de instrumentos específicos para medir resiliência em contexto laboral. Por outro lado, no que se refere à Escala de Resiliência utilizada por este trabalho, não há registros de seu uso em amostra de trabalhadores, sendo apenas encontrado seu uso em amostra de adolescentes escolares, que se refere ao estudo de adaptação transcultural o qual, como exposto, apresentou algumas inconsistências no que tange à AFE e sua decorrente estrutura fatorial, dificultando as comparações possíveis entre os dois estudos. $\mathrm{O}$ fato de a presente pesquisa ter trazido a Escala de Resiliência para a esfera do trabalho, então, não deve ser desconsiderado.

Com relação aos índices de fidedignidade, o alfa de Cronbach e a confiabilidade composta encontrados no presente estudo, apontaram que a escala apresentou bons indicadores, demonstrando ser uma medida consistente para avaliar resiliência em trabalhadores (VALENTINI; DAMÁSIO, 2016). Dados semelhantes também foram 
observados no estudo desenvolvido pelos autores americanos que construíram a escala originalmente $(\alpha=0,91)$, e os autores que realizaram o estudo de adaptação transcultural para o Brasil $(\alpha=0,80)$.

No que se refere aos escores médios em cada fator, observa-se que, tanto no fator Autodeterminação, quanto no fator Autoeficácia, a amostra alcançou uma pontuação que a localiza entre as alternativas de resposta "Concordo um pouco" e "Concordo", demonstrando valores indicativos de resiliência por parte desta. Dessa forma, pode-se inferir que a amostra, na presente pesquisa, não apresenta sinais de adoecimento no que tange aos aspectos de resiliência, portanto, não indica demanda de intervenção psicológica. Nesse sentido, a literatura aponta que a capacidade de manter bom funcionamento, mesmo em situações adversas, não é uma exceção, pelo contrário, é muito mais comum que as pessoas apresentem comportamentos adaptativos, mantendo-se saudáveis em circunstâncias desfavoráveis (LOPES; MARTINS, 2011). Embora a amostra não tenha apresentado uma demanda clínica, o presente estudo contribui, significativamente, para fins de diagnóstico, intervenção e pesquisa científica.

\section{CONSIDERAÇõES FINAIS}

O conceito de resiliência é relativamente novo no campo da Psicologia e vem sendo bastante discutido do ponto de vista teórico e metodológico pela comunidade científica. $\mathrm{O}$ termo se refere a processos que explicam a superação e adaptação positiva frente às adversidades que indivíduos, grupos ou organizações podem vivenciar.

De modo geral, os estudos empíricos brasileiros sobre resiliência têm demonstrado ligeira preferência pelos métodos qualitativos. No entanto, os estudos quantitativos vêm apontando resultados importantes, embora não haja consenso sobre a melhor forma de mensurar ou avaliar esse fenômeno, uma vez que a área possui ainda muita inconsistência conceitual que se reflete nas medidas existentes (LOPES; MARTINS, 2011). No contexto organizacional e do trabalho, essa problemática é ainda maior, uma vez que foram localizados poucos estudos validados no Brasil com trabalhadores (CARVALHO et al., 2014; HJEMDAL et al., 2009; LOPES; MARTINS, 2011). Desse modo, o presente estudo teve o objetivo de validar, em 
amostra de trabalhadores, a Escala de Resiliência, adaptada para o Brasil por Pesce et al. (2005).

Neste estudo, foram encontrados dois fatores, denominados "Autodeterminação" e "Autoeficácia". Autodeterminação foi entendido como um conjunto de comportamentos e habilidades que dotam o sujeito da capacidade de ser agente causal em relação ao cumprimento de seus objetivos e metas estabelecidas. Enquanto que o fator Autoeficácia foi definido como percepção do sujeito acerca de sua competência pessoal na resolução de tarefas e situações. Esses resultados não foram semelhantes aos obtidos por Pesce et al. (2005), que encontraram em sua estrutura fatorial três fatores, com ideias de resolução de ações e valores; independência e determinação e; indicativos de autoconfiança e capacidade de adaptação a situações. Não obstante, a estrutura proposta por este estudo apresenta-se conceitualmente clara e com propriedades psicométricas atestadas.

Cumpre ressaltar que o caráter inédito deste estudo decorre do fato de que há poucas investigações acerca da resiliência no contexto do trabalho. Portanto, o presente estudo apresenta à comunidade de estudiosos e profissionais da área aplicada um instrumental válido e fidedigno para uso em outras pesquisas e intervenções, trazendo mais esclarecimentos sobre o fenômeno resiliência no contexto de trabalho. No entanto, este estudo ainda não preenche a lacuna que existe em relação à ausência de instrumentos específicos de resiliência para o contexto laboral, pois se propôs a validar uma escala geral de resiliência em amostra de trabalhadores.

Os resultados deste estudo contribuem, ainda, para a área organizacional e do trabalho, na qual o tema da resiliência se insere em um contexto marcado por mudanças no ambiente de trabalho, incluindo fusões, privatizações, terceirização, entre outros aspectos do mundo contemporâneo do trabalho. Hoje, exige-se do trabalhador diversos tipos de adaptação, como a dependência da tecnologia e a flexibilidade de atividades e competências. Essa perspectiva tem impulsionado pesquisadores e profissionais a buscar formas de promover saúde e bem-estar no ambiente de trabalho (GOMIDE JÚNIOR; SILVESTRIN; OLIVEIRA, 2015). Assim, os estudos sobre resiliência no contexto de trabalho são recentes e vêm crescendo apenas nos últimos anos. 
A partir dos resultados encontrados nesse estudo, sugere-se às futuras pesquisas que avancem no sentido de preencher as lacunas existentes referentes à ausência de aparato científico específico de resiliência no trabalho. Por outro lado, destaca-se que estudos com outros propósitos podem ser conduzidos a partir deste, que disponibiliza um instrumento com propriedades psicométricas atestadas, para fins diagnósticos, intervencionistas e com vistas a promover resiliência no trabalho. Cabe destacar que os resultados apresentados neste estudo fazem parte de uma agenda de pesquisa maior, de modo que as estratégias para coleta de dados vêm sendo refinadas para sanarem as questões identificadas quanto à heterogeneidade da amostra de trabalhadores, a fim de realizar um estudo de validade mais aprofundado, com uso de Análise Fatorial Confirmatória (AFC).

$\mathrm{O}$ enfoque dado à resiliência no ambiente de trabalho representa uma mudança de perspectiva, deslocando-se da fraqueza do trabalhador em direção ao entendimento de sua capacidade de enfrentamento de situações adversas. No entanto, é preciso atentar para os possíveis desdobramentos decorrentes desse enfoque, para não cair em uma armadilha de exigência de produção de trabalhadores essencialmente resilientes diante das condições adversas do trabalho no mundo contemporâneo, tornando uma necessidade o perfil de trabalhador capaz de lidar com todas as exigências, pressionando-o a possuir uma atitude diferenciada que o destaque dos demais, uma atitude resiliente no ambiente laboral, enquanto as organizações contratantes desresponsabilizam-se da necessidade de oferecer ambientes e condições favoráveis para o cumprimento da atividade laboral. Em oposição, entendemos que o processo de resiliência deve atuar como um suporte adicional, defendendo os trabalhadores contra o estresse e o desenvolvimento negativo para sua saúde; em suma, atuar como elemento promotor de bem-estar e qualidade de vida no trabalho.

\section{REFEREÊNCIAS}

APPEL-SILVA, M.; WENDT, G. W.; ARGIMON, I. I. L. A teoria da autodeterminação e as influências socioculturais sobre a identidade. Psicologia em Revista, v. 16, n. 2, p. 351-369, 2010.

BARLACH, L.; LIMONGI-FRANÇA, A. C.; MALVEZZI, S. O conceito de resiliência aplicado ao trabalho nas organizações. Interamerican Journal of Psychology, v. 42, n. 1, p. 101-112, 2008. 
BELANCIERI, M. de F. et al. Promoção do processo de resiliência em enfermeiras: uma possibilidade? 2007. Tese de doutorado não-publicada, Pontifícia Universidade Católica de São Paulo, São Paulo. 2007.

BRANDÃO, J. M.; MAHFOUD, M.; GIANORDOLI-NASCIMENTO, I. F. A construção do conceito de resiliência em psicologia: discutindo as origens. Paidéia, v. 21, n. 49, p. 263-271, 2011.

CARVALHO, V. D.; TEODORO, M.; BORGES, L. O. Resiliência no contexto de trabalho. Em: PUENTE-PALACIOS, Katia; PEIXOTO, Adriano de Lemos Alves. Ferramentas de Diagnóstico para Organizações e Trabalho: Um Olhar a partir da Psicologia. Artmed Editora, 2015.

CARVALHO, V. D.; TEODORO, M. L. M.; BORGES, L. de O. Escala de Resiliência para Adultos: aplicação entre servidores públicos. Avaliação Psicológica, v. 13, n. 2, p. 287-295, 2014.

CASTLEDEN, M.; MCKEE, M.; MURRAY, V.; LEONARDI, G. Resilience thinking in health protection. Journal of Public Health, v. 33, n. 3, p. 369-377, 2011.

CATTELL, R. B. The scree test for the number of factors. Multivariate behavioral research, v. 1, n. 2, p. 245-276, 1966.

CIMBALISTA, S. Subjetividade e resiliência: o cotidiano adverso do trabalho flexível. $X$ Encontro Nacional da ABET, 2010.

CIMBALISTA, S. Reflexões sobre o trabalho e a subjetividade de trabalhadores resilientes sob o sistema de produção flexível. Revista da FAE, v. 9, n. 2, 2006.

COLWELL, S. R. (2016). The composite reliability calculator. Technical Report, DOI: 10.13140 / RG.2.1.4298.088.

COUTO-OLIVEIRA, V. Vida de mulher: gênero, pobreza, saúde mental e resiliência. 2007. 274 f. Dissertação (Mestrado em Psicologia Clínica e Cultura)-Universidade de Brasília, 2007.

DAMÁSIO, B. F. Uso da análise fatorial exploratória em psicologia. Avaliação psicológica, v. 11, n. 2, p. 213-228, 2012.

DANCEY, C. P.; REIDY, J. Estatística sem matemática para psicologia. Penso Editora, 2013.

COSTA, C. R. B. S. F.; ASSIS, S.G. Fatores protetivos a adolescentes em conflito com a lei no contexto socioeducativo. Psicologia \& Sociedade, v. 18, n. 3, p. 74-81, 2006.

ESTANISLAU, C.; CASTRO, D.; VIEIRA, A. M.; RESCH, S. O mundo do trabalho visto no cinema: busca por significados no documentário peões. Revista Pensamento Contemporâneo em Administração, v. 6, n. 2, p. 33-49, 2012.

FERNANDES, C. M.; SIQUEIRA, M. M. M.; VIEIRA, A. M. Impacto da percepção de suporte organizacional sobre o comprometimento organizacional afetivo: o papel moderador da liderança. Revista Pensamento Contemporâneo em Administração, v. 8, n. 4, p. 140-162, 2014.

FILENGA, D.; VIEIRA, A. M. Notas sobre o trabalho e seu contexto social. Revista UNIABEU, v. 5, p. 1-16, 2012.

FONTES, A. P.; AZZI, R. G. Crenças de autoeficácia e resiliência: apontamentos da literatura sociocognitiva. Estudos de Psicologia (Campinas), 2012. 
GOMIDE JÚNIOR, S.; SILVESTRIN, L. H. B.; OLIVEIRA, Á. de F. Bem-estar no trabalho: o impacto das satisfações com os suportes organizacionais e o papel mediador da resiliência no trabalho. Revista Psicologia Organizações e Trabalho, v. 15, n. 1, p. 19-29, 2015.

GURGEL, L. G. et al. Avaliação da resiliência em adultos e idosos: revisão de instrumentos. Estudos de Psicologia (Campinas), v. 30, n. 4, p. 487-496, 2013.

HJEMDAL, $\mathrm{O}$ et al. Exploring the psychometric properties of the resilience scale for adults in a Brazilian sample. Facet new horizons in theory construction and data analysis, p. 120-138, 2009.

HORN, J. L. A rationale and test for the number of factors in factor analysis. Psychometrika, v. 30, n. 2, p. 179-185, 1965.

JUNQUEIRA, M. de F. P. da S.; DESLANDES, S. F. Resiliência e maus-tratos à criança. Cad. Saúde Pública, Rio de Janeiro, v. 19, n. 1, p. 227-235, fev. 2003.

KAISER, H. F. The application of electronic computers to factor analysis. Educational and psychological measurement, v. 20, n. 1, p. 141-151, 1960.

LIBÓRIO, R. M. C.; CASTRO, B. M.; COELHO, A. E. L. Desafios metodológicos para a pesquisa em resiliência: conceitos e reflexões críticas. In: AGLIO, D. D. D.; KOLLER, S. H.; YUNES, M. A. M. Resiliência e Psicologia Positiva: Interfaces do Risco à Proteção. São Paulo: Casa do Psicólogo, 2006. p. 89-115

LOPES, V. R.; MARTINS, M. do C. F. Validação fatorial da escala de resiliência de Connor-Davidson (Cd-Risc-10) para brasileiros. Revista Psicologia Organizações e Trabalho, v. 11, n. 2, p. 36-50, 2011.

LUTHAR, S. S.; CICCHETTI, D.; BECKER, B. The construct of resilience: A critical evaluation and guidelines for future work. Child development, v. 71, n. 3, p. 543-562, 2000.

MALVEZZI, S. A construção da identidade profissional no modelo emergente de carreira. Organizações \& Sociedade, v. 7, n. 17, p. 137-143, 2000.

MARTINS, M. C. F. Resiliência no Trabalho. In: BENDASSOLI, P. F.; BORGES-ANDREADE, J. E. Dicionário de Psicologia do Trabalho e das Organizações. Nacional: Casa do Psicólogo, 2015. p. 581-587.

ONÇA, S. da S. Resiliência em trabalhadores: impacto da auto-eficácia e da percepção de suporte social. 2011. 171 f. Dissertação (Mestrado em Psicologia). Programa de Pós-graduação em Psicologia da Saúde, Universidade Metodista de São Paulo, São Paulo. 2011.

PESCE R. P., ASSIS S. G., AVANCI J. Q., S. N. C., MALAQUIAS J. V., CARVALHAES R. Adaptação transcultural, confiabilidade e validade da escala de resiliência. Cad Saúde Pública, p. 436-448, 2005.

PIACENTINI, N. Construção e estudos iniciais de validação de uma medida de resiliência. 2014. 119 f. Dissertação (Mestrado em Psicologia). Programa de Pós-graduação em Psicologia, Universidade Federal de Santa Catarina, Santa Catarina. 2014.

POLETTO, M. Contextos ecológicos de promoção de resiliência para crianças e adolescentes em situação de vulnerabilidade. 2007. 104 f. Dissertação (Mestrado em Psicologia). Instituto 
de Psicologia, Universidade Federal do Rio Grande do Sul, Rio Grande do Sul. 2007.

POLETTO, M.; KOLLER, S. H. Contextos ecológicos: promotores de resiliência, fatores de risco e de proteção. Estudos de psicologia, v. 25, n. 3, p. 405-416, 2008.

POLETTO, M.; KOLLER, S. H. Resiliência: uma perspectiva conceitual e histórica. Resiliência e psicologia positiva: Interfaces do risco à proteção, p. 19-44, 2006.

RIBEIRO, A. C. de A. et al . Resiliência no trabalho contemporâneo: promoção e/ou desgaste da saúde mental. Psicologia em estudo, v. 16, n. 4, p. 623-633, 2011.

SANTOS, M. M. O.; QUEL, L. F.; VIEIRA, A. M.; ROSINI, A. M. Indicadores de Desempenho e Engajamento Profissional em Organizações Inovadoras. Revista de Gestão e Secretariado, v. 10, n. 1, p. 192-212, 2019.

SOUZA, M. T. S. de; CERVENY, C. M. de O. Resiliência psicológica: revisão da literatura e análise da produção científica. Interamerican journal of psychology, v. 40, n. 1, p. 115-122, 2006.

WAGNILD, G.; YOUNG, H. Development and psychometric. Journal of nursing measurement, v. 1, n. 2, p. 165-178, 1993.

YUNES, M. A. M. Psicologia positiva e resiliência: o foco no indivíduo e na família. Psicologia em estudo, v. 8, n. 1, p. 80-95, 2003.

YUNES, M. A. M.; MENDES, N. F.; ALBUQUERQUE, B. de M. Percepções e crenças de agentes comunitários de saúde sobre resiliência em família monoparentais pobres. Texto contexto - enferm., Florianópolis, v. 14, n. spe, p. 24-31, 2005.

YUNES, M. A. M.; SZYMANSKI, H.; TAVARES, J. Resiliência: noção, conceitos afins e considerações críticas. Resiliência e educação, v. 2, p. 13-43, 2001.

Recebido em: 31-1-2018

Aprovado em: 1-6-2019

Avaliado pelo sistema double blind review.

Disponível em http://mjs.metodista.br/index.php/roc 